\title{
Exact computation of maximum induced forest
}

\author{
Igor Razgon * \\ Computer Science Department, University College Cork, Ireland \\ i.razgon@cs.ucc.ie
}

\begin{abstract}
We propose a backtrack algorithm that solves a generalized version of the Maximum Induced Forest problem (MIF) in time $O^{*}\left(1.8899^{n}\right)$. The MIF problem is complementary to finding a minimum Feedback Vertex Set (FVS), a well-known intractable problem. Therefore the proposed algorithm can find a minimum FVS as well. To the best of our knowledge, this is the first algorithm that breaks the $O^{*}\left(2^{n}\right)$ barrier for the general case of FVS. Doing the analysis, we apply a more sophisticated measure of the problem size than the number of nodes of the underlying graph.
\end{abstract}

\section{Introduction}

Exact exponential algorithms are techniques for solving intractable problems with better complexity than trivial brute-force exploring of all the possible combinations. Examples of such algorithms include: [9] for maximum independent set, [1] for chromatic number, [3] for 3-COLORABILITY, [2] for 3-SAT, [4] for dominating set, and others. A recent overview of exact algorithms is provided in [10].

In this paper we propose an $O^{*}\left(1.8899^{n}\right)$ exact algorithm for solving the following problem. Given a graph $G$ and a subset $K$ of its vertices, find a largest superset $S$ of $K$ such that the subgraph of $G$ induced by $S$ is acyclic. If $K=$ $\emptyset$ then $S$ is a Maximum Induced Forest (MIF) of $G$. The complement of $S$, $V(G) \backslash S$, is a minimum Feedback Vertex Set (FVS) of $G$, i.e. a set of vertices that participate in all the cycles of $G$. Computing a minimum FVS is a "canonical" intractable optimization problem, whose NP-complete version is mentioned in [7]. To the best of our knowledge, the proposed algorithm is the first that breaks the $O^{*}\left(2^{n}\right)$ barrier for the general case of FVS. Previous studies $[6,8]$ describe exact algorithms only for special cases of FVS.

The proposed algorithm computes MIF using the "branch-and-prune" strategy ([10], Section 4). Using this strategy for computing of MIF is not straightforward. The reason is that selection of a new vertex for MIF does not necessarily cause additional pruning: a vertex can be pruned only if it induces a cycle with the "already selected" vertices. For graphs with a large girth, many vertices must be selected before at least one can be discarded.

\footnotetext{
* I would like to thank Fedor Fomin, who inspired me to investigate the problem, and an anonymous reviewer who suggested me a way of improving the result reported in the first version of the paper.
} 
To overcome this difficulty, we analyze complexity of the algorithm by applying a more sophisticated measure than the number of vertices of the residual graph, a strategy suggested in [5]. In particular, we observe that all the "remaining" vertices can be partitioned into the vertices that have neighbours with the already selected vertices and the vertices that do not have them. We associate the vertices of the former class with weight 1 and the vertices of the latter class with weight 1.565 , a constant guessed by a computational procedure. The proposed measure is the sum of weights of all the vertices of the residual graph. Further analysis yields an upper bound $O^{*}\left(1.50189^{m}\right)$, where $m$ is the value of the applied measure for the input graph $G$. We then demonstrate that $m \leq 1.565 n$, where $n$ is the number of vertices of $G$, which results in an upper bound of $O^{*}\left(\left(1.50189^{1.565}\right)^{n}\right)$. Taking into account that $1.8898<1.50189^{1.565}<1.8899$, this bound is transformed to $O^{*}\left(1.8899^{n}\right)$ by rounding.

The rest of the paper is organized as follows. Section 2 introduces the necessary terminology. Section 3 presents the proposed algorithm. Section 4 proves correctness of the algorithm and provides complexity analysis ${ }^{1}$.

\section{Preliminaries}

A simple undirected graph is referred in this paper as a graph. A set of vertices of a graph $G$ is denoted by $V(G)$. Given $S \subseteq V(G)$, we denote by $G[S]$ the subgraph of $G$ induced by $S$ and by $G \backslash S$ the subgraph of $G$ induced by $V(G) \backslash S$. If $S$ consists of a single vertex $v$, we write $G \backslash v$ rather than $G \backslash\{v\}$. Two vertices $v$ and $w$ of $G$ are $S$-connected if they are adjacent or if there is a path $v, p_{1}, \ldots, p_{m}, w$, where $\left\{p_{1}, \ldots p_{m}\right\} \subseteq S$.

The set $S$ is a maximum induced forest (MIF) if $G[S]$ is acyclic and $S$ is the largest set subject to this property ${ }^{2}$. In addition, we introduce the notion of a T-MIF.

Definition 1. Let $T \subseteq V(G)$. A T-MIF of $G$ is a largest superset $S$ of $T$ such that $G[S]$ is acyclic.

Clearly, the definition makes sense only when $G[T]$ is acyclic. Observe that a $\emptyset$-MIF of $G$ is an ordinary MIF.

To present complexity of algorithms, we use the $O^{*}$ notation [10], which suppresses the polynomial factor. For example, $O\left(n^{2} 2^{n}\right)$ is written as $O^{*}\left(2^{n}\right)$.

\section{The algorithm}

In this section we present an algorithm for computing a MIF of a given graph. We start with extending our notation.

Let $G$ be a graph and let $T$ be a subset of its vertices. We recognize the following classes of vertices of $G \backslash T$.

\footnotetext{
${ }^{1}$ Due to space constraints, proofs of some technical lemmas are omitted.

${ }^{2}$ It is more convenient for us to represent a MIF as a set of vertices rather than a subgraph of $G$.
} 
- Boundary vertices denoted by $\operatorname{Bnd}(G, T)$. The set $\operatorname{Bnd}(G, T)$ contains all vertices $v \in V(G \backslash T)$ such that $v$ is adjacent to exactly one vertex of $T$.

- Conflicting vertices denoted by $\operatorname{Cnf}(G, T)$. The set $\operatorname{Cnf}(G, T)$ contains all vertices $v \in V(G \backslash T)$ such that $v$ is adjacent to at least two vertices of the same connected component of $G[T]$.

- Free vertices denoted by $\operatorname{Free}(G, T)$. The set $\operatorname{Free}(G, T)$ contains all vertices $v \in V(G \backslash T)$ such that $v$ is not adjacent to any vertex of $T$.

Now we are ready to introduce the algorithm Main_MIF (Algorithm 1). It gets as input a graph $G$ and a subset $K$ of $V(G)$. The algorithm returns (as we will prove further) a $K$-MIF of $G$. Clearly, setting $K$ to $\emptyset$ will make the algorithm to return a MIF of $G$.

The algorithm Main_MIF starts with checking whether $G[K]$ is acyclic. If not, $F A I L$ is returned immediately (line 1 of Algorithm 1 ) because no $K$ MIF of $G$ exists in this case. Otherwise, the function Find_MIF runs (line 2 of Algorithm 1).

Function Find_MIF is the main "search engine" of Main $M I F$. It is described in lines 3-31 of Algorithm 1. The function gets as input a graph $G_{1}$, and subsets $T_{1}$ and $K_{1}$ of $V\left(G_{1}\right)$. The function is supposed to return a $T_{1} \cup K_{1}$-MIF of $G_{1}$ (provided that $G_{1}\left[T_{1} \cup K_{1}\right]$ is acyclic).

If $T_{1} \cup K_{1}=V\left(G_{1}\right)$, Find_MIF returns $T_{1} \cup K_{1}$ (lines 4 and 5 of Algorithm 1). Otherwise, the execution can be divided into four stages: selecting a vertex $v$ of $V\left(G_{1}\right) \backslash\left(T_{1} \cup K_{1}\right)$, a recursive call processing the case when $v$ is added to $T_{1} \cup K_{1}$, a recursive call processing the case when $v$ is eliminated from $G_{1}$, and returning the maximum-size set among the ones returned by the above two recursive calls.

Selection of a vertex $v$ is described in lines 7-11 of Algorithm 1. The vertex is taken from $\operatorname{Bnd}\left(G_{1}, T_{1}\right)$ unless the set is empty. In this case, the function selects an arbitrary vertex that does not belong to $T_{1} \cup K_{1}$.

Having selected a vertex $v$, the function adds it to $T_{1} \cup K_{1}$ (line 12 of Algorithm 1). The addition is performed by function T_Update (Algorithm $2)$. Applying of $T_{-} U p d a t e$ in line 12 returns a triplet $\left(G_{2}, T_{2}, K_{2}\right)$, in which $T_{2} \cup K_{2}=T_{1} \cup K_{1} \cup\{v\}, v$ itself and all vertices of $K_{1}$ that are $K_{1}$-connected to $v$ are "moved" to $T_{2}, G_{2}$ is obtained from $G_{1}$ by removing all vertices of $C n f\left(G_{1}, T_{2}\right)$ because every one of them induces cycles being added to $T_{2}$. Function Find_MIF is applied recursively to $\left(G_{2}, T_{2}, K_{2}\right)$ in line 13 and returns a set $S_{2}$.

The way a vertex $v$ is selected and then added to $T_{2} \cup K_{2}$ ensures that the inputs $\left(G^{\prime}, T^{\prime}, K^{\prime}\right)$ of all recursive applications of FindIndep have a number of invariant properties which are crucial for our analysis (Section 4). Two most important properties are that any connected component of $G^{\prime}$ contains at most one connected component of $G^{\prime}\left[T^{\prime}\right]$, and that there are no edges between vertices of $T^{\prime}$ and vertices of $K^{\prime}$.

Processing the case, where $v$ is eliminated from $G_{1}$ (lines 14-29 of Algorithm $1)$, depends on the number of vertices of $V\left(G_{1}\right) \backslash\left(T_{1} \cup K_{1} \cup\{v\}\right)$ that are $K_{1^{-}}$ connected to $v$. If there is at most one such vertex, the function decides that 
$S_{2}$ is a $T_{1} \cup K_{1}$-MIF of $G_{1}$ and returns it (lines 15-16 of Algorithm 1). The case when there are exactly 2 such vertices is processed in lines 17-25. The set $W$ of these two vertices is added to $T_{1} \cup K_{1}$ by function $K_{-} U p d a t e$ (Algorithm 3 ). This function returns $F A I L$ if $G_{1}\left[T_{1} \cup K_{1} \cup W\right]$ contains cycles. In this case, Find_MIF returns $S_{2}$. Otherwise, Find_MIF is applied recursively to the triplet returned by $K_{-} U p d a t e$, returns a set $S_{3}$, and the largest set among $S_{2}$ and $S_{3}$ is returned in line 22 . If the number of vertices of $V\left(G_{1}\right) \backslash\left(T_{1} \cup K_{1} \cup\{v\}\right)$ that are $K_{1}$-connected to $v$ is at least 3, Find_MIF returns the largest set among $S_{2}$ and $S_{3}$, where $S_{3}$ is returned by the recursive application of Find $\triangle I F$ to $\left(G_{1} \backslash v, T_{1}, K_{1}\right)$ (lines 26-28 of Algorithm 1).

Consider the intuition behind the decisions made by the algorithm in lines 1525 . For this purpose, assume that $K_{1}=\emptyset$. That is, we consider the cases where $v$ have 1 or 2 neighbours that are not in $T_{1}$. In the former case, let $w \in V\left(G_{1}\right) \backslash T_{1}$ be the considered neighbour of $v$. Observe that it is safe to add $v$ to $T_{2}$. Really, any $T_{1}$-MIF $S$ of $G_{1}$ that does not contain $v$ has to contain $w$ (otherwise, we get contradiction to the maximality of $S$ ). In this case replacing $w$ by $v$ in $S$, we get another $T_{1}$-MIF of $G_{1}$.

Assume now that $v$ is adjacent to vertices $w_{1}, w_{2} \in V\left(G_{1}\right) \backslash T_{1}$ and that $v$ does not belong to any $T_{1}$-MIF of $G_{1}$. Then, any $T_{1}$-MIF of $G_{1}$ contains both $w_{1}$ and $w_{2}$, otherwise, arguing as for the previous case, we get a contradiction with our assumption. A subtle question is where to add $w_{1}$ and $w_{2}$. The point is that the invariant property that every component of $G_{1}$ contains at most one component of $G_{1}\left[T_{1}\right]$ should not be violated for the inputs of the subsequent recursive calls of the Find_MIF function. To satisfy this requirement, for example, when none of $w_{1}$ and $w_{2}$ have neighbours in $T_{1}$, the function $K_{-} U p d a t e$ adds them to $K_{2}$, not to $T_{2}$. That is, even if $K=\emptyset$ in the original input, it can be transformed to a nonempty set in one of subsequent recursive calls of Find_MIF. Thus the necessity to handle the case when $v$ is adjacent to exactly two "remaining" vertices is what caused the author to consider a generalized version of the MIF-problem.

\section{Analysis}

The analysis of the Main_MIF algorithm is organized as follows. In Section 4.1 we introduce the notion of a Fair Configuration (FC) and prove a number of properties of FCs. In Section 4.2 we define a search tree generated by function Find_MIF with the nodes corresponding to the inputs of the recursive calls of Find_MIF. We prove that all these inputs are FCs. Then, based on properties of FCs, we prove correctness of Main_MIF (section 4.3) and analyze its complexity (section 4.4). Due to space constraints, proofs of some technical lemmas are omitted.

\subsection{Fair configurations and their properties}

Definition 2. Let $G$ be a graph and let $T$ and $K$ be subsets of $V(G)$. A triplet $(G, T, K)$ is a Fair Configuration $(F C)$ if the following conditions hold: 


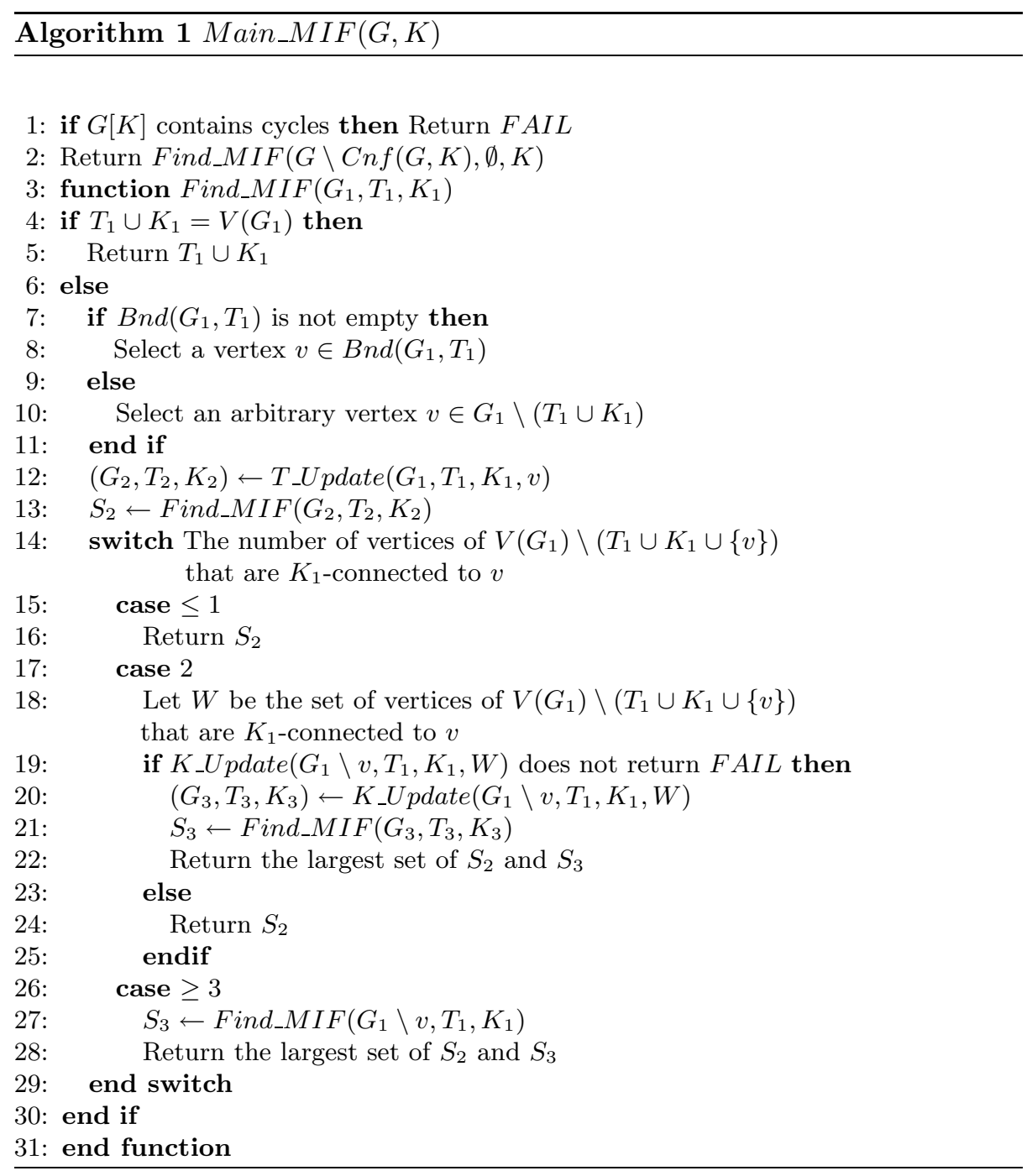

\begin{tabular}{l}
\hline Algorithm $2 T \_U p d a t e(G, T, K, v)$ \\
\hline \\
1: Let $S$ be the subset of vertices of $K$ that are $K$-connected to $v$ \\
2: $T^{\prime} \leftarrow T \cup\{v\} \cup S$ \\
3: $K^{\prime} \leftarrow K \backslash(\{v\} \cup S)$ \\
4: $G^{\prime} \leftarrow G \backslash C n f\left(G, T^{\prime}\right)$ \\
5: $\operatorname{Return}\left(G^{\prime}, K^{\prime}, T^{\prime}\right)$ \\
\hline
\end{tabular}




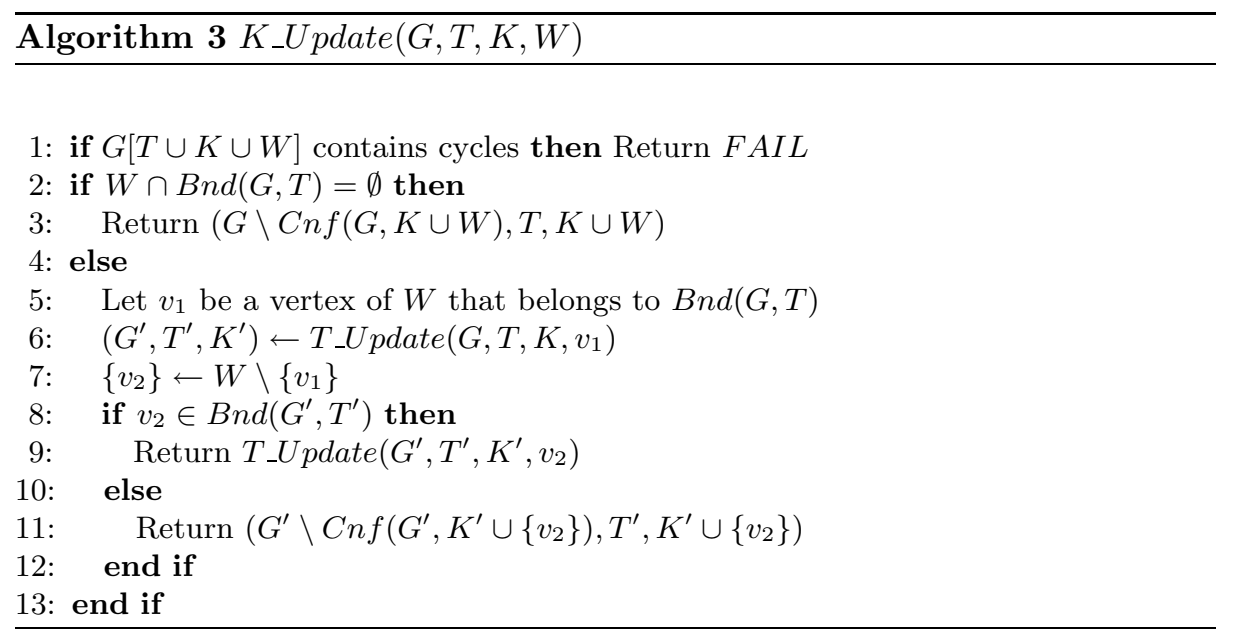

- $K \subseteq \operatorname{Free}(G, T)$;

- $G[T]$ and $G[K]$ are acyclic;

- $\operatorname{Cnf}(G, T)=\operatorname{Cnf}(G, K)=\emptyset$;

- every connected component of $G$ contains at most one connected component of $G[T]$.

Due to importance of the notion for the proposed analysis, we demonstrate it on an example.

Let $G$ be the graph shown in Figure 1, the black circles represent the vertices of $T$, the crossed circles represent the vertices of $K$, the other vertices are represented by the white circles. Observe that $(G, T, K)$ is a FC. Indeed, there are no edges between the vertices of $T$ and $K$, both $T$ and $K$ induce acyclic subgraphs of $G$, no single vertex of $V(G) \backslash(T \cup K)$ makes cycles with $T$ and $K$. Finally, every connected component of $G$ contains at most one connected component of $G[T]$. Note that by definition of a FC, the last requirement is not necessary for $G[K]$. In our example, the component induced by vertices $v_{9}$ to $v_{12}$ contains two components of $G[K]$.

Lemma 1. Let $(G, T, K)$ be a FC. Then $T \cup \operatorname{Bnd}(G, T) \cup \operatorname{Free}(G, T)=V(G)$. 3

Lemma 2. Let $(G, T, K)$ be a $F C$ and let $v \in V(G) \backslash(T \cup K)$. Assume that one of the following properties holds:

$-v \in \operatorname{Bnd}(G, T)$;

$-\operatorname{Bnd}(G, T)=\emptyset$.

Then $\left(G^{\prime}, T^{\prime}, K^{\prime}\right)=T_{\_} U$ pdate $(G, T, K, v)$ is a $F C$.

\footnotetext{
${ }^{3}$ In other words, any vertex of $V(G) \backslash T$ is adjacent to at most one vertex of $T$.
} 

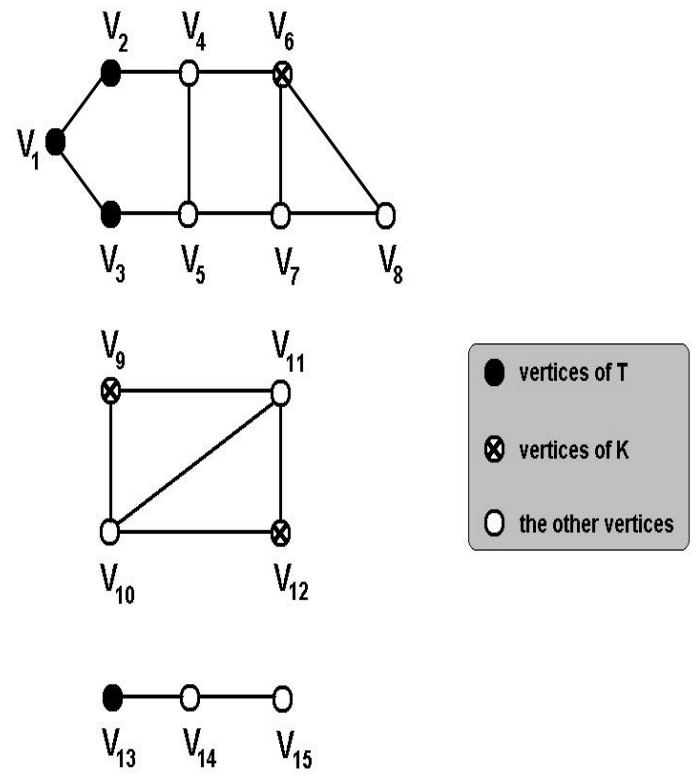

Fig. 1. A Fair Configuration (FC)

As a result of application of $T_{-} U p d a t e$, some vertices change their "roles". This statement is described precisely in the following lemma.

Lemma 3. Let $(G, T, K)$ be a $F C$ and let $v \in V(G)$ be a vertex such that $\left(G^{\prime}, T^{\prime}, K^{\prime}\right)=T \_U p d a t e(G, T, K, v)$ is a $F C$.

Let $w \in V(G) \backslash(T \cup K \cup\{v\})$ be a vertex, which is $K$-connected to $v$. Then

- $w \notin \operatorname{Free}\left(G^{\prime}, T^{\prime}\right)$;

- in addition, if $w \in \operatorname{Bnd}(G, T)$ then $w \notin V\left(G^{\prime}\right)$.

Lemma 4. Let $(G, T, K)$ be a FC. Let $S \subseteq V(G) \backslash(T \cup K)$ and $W \subseteq F r e e(G, T) \backslash$ $K$ be two disjoint sets. Then $(G \backslash S \backslash C n f(G \backslash S, K \cup W), T, K \cup W)$ is a $F C$.

\subsection{The search tree $S T$}

In this section we define a search tree $S T$ explored by Main_MIF. The root of the tree is associated with a triplet $(G \backslash C n f(G, K), \emptyset, K)$, where $G$ and $K$ constitute the input of Main_MIF. Assume that a node $x$ of $S T$ is associated with a triplet $\left(G_{1}, T_{1}, K_{1}\right)$. The structure of the subtree rooted by $x$ depends on the execution of Find_MIF $\left(G_{1}, T_{1}, K_{1}\right)$. If $T_{1} \cup K_{1}=V\left(G_{1}\right)$ then $x$ is a leaf. Otherwise, $x$ has a child associated with the triplet returned by $T_{-} \operatorname{Update}\left(G_{1}, T_{1}, K_{1}, v\right)$, where $v$ is the vertex selected by Find_MIF $\left(G_{1}, T_{1}, K_{1}\right)$ in lines 7-11 of Algorithm 1. It is the only child if Find_MIF $\left(G_{1}, T_{1}, K_{1}\right)$ executes line 16 or line 24. If line 22 is executed then $x$ has the additional child associated with 
$K_{-} U p d a t e\left(G_{1} \backslash v, T_{1}, K_{1},\left\{v_{1}, v_{2}\right\}\right)$; if line 28 is executed, the additional child is associated with the triplet $\left(G_{1} \backslash v, T_{1}, K_{1}\right)$.

Lemma 5. ST is of finite size.

Lemma 6. The triplet associated with every node of $S T$ is a FC.

\subsection{Correctness Proof.}

In this section we will prove correctness of Main_MIF by demonstrating that Main_MIF $(G, K)$ returns a $K$-MIF of $G$.

Lemma 7. Let $(G, T, K)$ be a $F C$ and let $v \in V(G) \backslash(T \cup K)$. Let $S$ be a $T \cup K-M I F$ of $G$. Then either $v \in S$ or any cycle in $G[S \cup\{v\}]$ involves a vertex $w \in V(G) \backslash(T \cup K \cup\{v\})$, which is K-connected to $v$.

Proof. Assume that $v \notin S$ and let $v, v_{1}, \ldots v_{m}$ be a cycle of $G[S \cup\{v\}]$ (clearly, $v$ participates in any cycle of $G[S \cup\{v\}]$ because $G[S]$ is acyclic). If either $v_{1}$ or $v_{m}$ belongs to $V(G) \backslash(T \cup K)$, we are done. Otherwise, note that $\left\{v_{1}, v_{m}\right\} \nsubseteq T$ because $v$ cannot be connected to more than one vertex of $T$, by Lemma 1 . It follows also that $\left\{v_{1}, \ldots, v_{m}\right\} \nsubseteq K$ because the opposite would mean that $v \in \operatorname{Cnf}(G, K)$. Assume without loss of generality that $v_{1} \in K$. Let $i$ be the smallest index such that $v_{i} \notin K$, while $v_{i-1} \in K$. Note that by definition of a FC $v_{i} \notin T$ (because existence of an edge between $K$ and $T$ would follow otherwise). Thus $v_{i} \in V(G) \backslash(T \cup K)$ and the path $v, v_{1}, \ldots, v_{i}$, all intermediate vertices of which belong to $K$, certifies that $v_{i}$ is $K$-connected to $v$.

Lemma 8. Let $G$ be a graph, $T \subset V(G)$ such that $G[T]$ is acyclic, and $U \subseteq$ $V(G) \backslash T$. Assume that no T-MIF of $G$ intersects with $U$. Then any T-MIF of $G \backslash U$ is a $T$-MIF of $G$.

Lemma 9. Let $(G, T, K)$ be a $F C$ and let $v \in V(G) \backslash(T \cup K)$. Let $\left(G^{\prime}, T^{\prime}, K^{\prime}\right)=$ $T \_U p d a t e(G, T, K, v)$. Assume that at least one $T \cup K$-MIF of $G$ contains $v$. Then any $T^{\prime} \cup K^{\prime}$-MIF of $G^{\prime}$ is a $T \cup K$-MIF of $G$.

Lemma 10. Let $(G, T, K)$ be a $F C$. Let $v \in V(G) \backslash(T \cup K)$ be a vertex, which is $K$-connected to at most one vertex of $G \backslash(T \cup K \cup\{v\})$. Let $\left(G^{\prime}, T^{\prime}, K^{\prime}\right)=$ $T \_U p d a t e(G, T, K, v)$. Then any $T^{\prime} \cup K^{\prime}-M I F$ of $G^{\prime}$ is a $T \cup K-M I F$ of $G$.

Proof. If at least one $T \cup K$-MIF of $G$ contains $v$, the statement follows from Lemma 9. Otherwise, let $S$ be a $T \cup K$-MIF of $G$. By Lemma 7, every cycle of $G[S \cup\{v\}]$ contains a vertex of $V(G) \backslash(T \cup K \cup\{v\})$, which is $K$-connected to $v$. By the condition of the lemma, there is at most one such a vertex, say, $w$. Therefore $w$ participates in all the cycles of $G[S \cup\{v\}]$, and removing of $w$ breaks all the cycles. Clearly, $S \cup\{v\} \backslash\{w\}$ is a $T \cup K$-MIF of $G$ containing $v$, in contradiction to our assumption. 
Lemma 11. Let $(G, T, K)$ be a $F C$. Let $v \in V(G) \backslash(T \cup K)$ be a vertex, which is $K$-connected to exactly two vertices $v_{1}$ and $v_{2}$ of $G \backslash(T \cup K \cup\{v\})$. Then at least one of the following two statements is true.

- Let $\left(G_{1}, T_{1}, K_{1}\right)=T_{-} U p d a t e(G, T, K, v)$. Then any $T_{1} \cup K_{1}-M I F$ of $G_{1}$ is a $T \cup K-M I F$ of $G$.

- K_Update $\left(G \backslash v, T, K,\left\{v_{1}, v_{2}\right\}\right)$ does not return FAIL. Moreover, let $\left(G_{2}, T_{2}, K_{2}\right)=K_{-} U p d a t e\left(G \backslash\{v\}, T, K,\left\{v_{1}, v_{2}\right\}\right)$.

Then any $T_{2} \cup K_{2}-M I F$ of $G_{2}$ is a $T \cup K$-MIF of $G$.

Proof. Assume that the first statement does not hold. By Lemma 9, no $T \cup K$-MIF of $G$ contains $v$. Let $S$ be a $T \cup K$-MIF of $G$. According to Lemma 7, any cycle in $G[S \cup\{v\}]$ involves a vertex of $G \backslash(T \cup K \cup\{v\})$, which is $K$ connected to $v$. If $S$ contains only one such vertex, say, $v_{1}$, then removing $v_{1}$ breaks all the cycles and $S \cup\{v\} \backslash\left\{v_{1}\right\}$ is a $T \cup K$-MIF of $G$ in contradiction to our assumption. It follows that $\left\{v_{1}, v_{2}\right\} \subseteq S$. Clearly, $S$ is a $T \cup K \cup\left\{v_{1}, v_{2}\right\}$-MIF of $G$ because otherwise we get a contradiction with being $S$ a $T \cup K$-MIF of $G$. It follows that any $T \cup K \cup\left\{v_{1}, v_{2}\right\}$-MIF of $G$ is a $T \cup K$-MIF of $G$. Then, by Lemma 8, any $T \cup K \cup\left\{v_{1}, v_{2}\right\}$-MIF of $G \backslash v$ is a $T \cup K$-MIF of $G$. Consequently $(G \backslash v)\left[T \cup K \cup\left\{v_{1}, v_{2}\right\}\right]$ is acyclic and hence $K_{-} U p d a t e\left(G, T, K,\left\{v_{1}, v_{2}\right\}\right)$ does not return FAIL. Furthermore, it follows from the description of $K_{-} U p d a t e$ that $T_{2} \cup K_{2}=T \cup K \cup\left\{v_{1}, v_{2}\right\}$ and $G_{2}$ is obtained from $G \backslash v$ by removing vertices that make cycles with $T \cup K \cup\left\{v_{1}, v_{2}\right\}$ in $G \backslash v$. Thus, any $T_{2} \cup K_{2}$-MIF of $G_{2}$ is a $T \cup K$-MIF of $G$ by Lemma 8 and the above reasoning.

Theorem 1. For any triplet $\left(G_{1}, T_{1}, K_{1}\right)$ associated with a node of $S T$, Find_MIF $\left(G_{1}, T_{1}, K_{1}\right)$ returns a $T_{1} \cup K_{1}$-MIF of $G_{1}$.

Proof. Let $x_{1}, x_{2}, \ldots$ be an order of nodes of $S T$ such that children are ordered before their parents; existence of such an order follows from Lemma 5 . The proof is by induction on the sequence. We also use the fact that the triplet associated with every node $x$ of $S T$ is a FC (Lemma 6).

Clearly, the statement holds for all leaves of $S T$ and, in particular, for $x_{1}$. Consider a non-leaf node $x_{i}$, assuming validity of the theorem for all nodes placed before, and denote the FC associated with $x_{i}$ by $\left(G_{1}, T_{1}, K_{1}\right)$. The FCs associated with the children of $x_{i}$ are exactly the inputs of the recursive calls performed by Find_MIF $\left(G_{1}, T_{1}, K_{1}\right)$. By the induction assumption, these recursive calls work properly.

If the vertex $v$ picked by Find_MIF $\left(G_{1}, T_{1}, K_{1}\right)$ is $K_{1}$-connected to exactly one vertex of $V\left(G_{1}\right) \backslash\left(T_{1} \cup K_{1} \cup\{v\}\right)$, the correctness follows from Lemma 10; in the case of two vertices, the correctness follows from Lemma 11; in the case of three or more vertices, the correctness follows from Lemmas 8 and 9.

Corollary 1. Let $G$ be a graph and $K \subseteq V(G)$. If $G[K]$ is acyclic, Main_MIF $(G, K)$ returns a $K$-MIF of $G$. 


\subsection{Complexity analysis}

In this section we analyse the complexity of Main_MIF by deriving the upper bound on the number of nodes of $S T$. For the complexity analysis, we associate with every node $x$ of $S T$ the measure $Y(x)=c \mid$ Free $\left(G_{1}, T_{1}\right) \backslash K_{1} \mid+$ $\left|\operatorname{Bnd}\left(G_{1}, T_{1}\right)\right|$, where $\left(G_{1}, T_{1}, K_{1}\right)$ is the triplet associated with $x, c=1.565$. In other words, the elements of Free $\left(G_{1}, T_{1}\right) \backslash K_{1}$ are assigned with weight $c$, the elements of $\operatorname{Bnd}\left(G_{1}, T_{1}\right)$ are assigned with weight $1, Y(x)$ is the sum of all the weights. The complexity analysis is structured as follows. For a given two nodes $x$ and $z$ such that $x$ is the parent of $z$, we evaluate $Y(x)-Y(z)$. Based on the evaluation, we obtain an upper bound on the number of nodes of the subtree rooted at $x$. This upper bound is $O^{*}\left(\alpha(c)^{Y(x)}\right)$, where $\alpha(c)$ is the constant depending on $c$. For $c=1.565, \alpha(c)=1.50189$. Then we notice that for the root node $r$, the value of $Y(r)$ is at most $c n$, where $n$ is the number of vertices of the original graph. Thus we obtain the upper bound $O^{*}\left(1.50189^{1.565 n}\right)$. Taking into account that $1.8898<1.50189^{1.565}<1.8899$, the upper bound obtained after rounding the base of the exponent is $O^{*}\left(1.8899^{n}\right)$.

The constant $c=1.565$ was guessed by a binary search computational procedure that explored the range from 1.005 to 2 by steps of 0.005 and for every considered constant $c$ computed $\alpha(c)^{c}$. The smallest value of this expression was obtained for $c=1.565$.

Lemma 12. Let $x$ be a non-leaf node of $S T$ associated with a triplet $\left(G_{1}, T_{1}, K_{1}\right)$, let a node $z$ associated with a triplet $\left(G_{2}, T_{2}, K_{2}\right)$ be a child of $x$, and let $v \in$ $V\left(G_{1}\right) \backslash\left(T_{1} \cup K_{1}\right)$. Then the following statements hold.

1. If $\left(G_{2}, T_{2}, K_{2}\right)=T_{-} U$ pdate $\left(G_{1}, T_{1}, K_{1}, v\right)$ then $Y(z) \leq Y(x)-((c-1)|W|+$ $1)$, where $W$ is the set of vertices of $V\left(G_{1}\right) \backslash\left(T_{1} \cup K_{1} \cup\{v\}\right)$ that are $K_{1}$ connected to $v$.

2. If $\left(G_{2}, T_{2}, K_{2}\right)=K_{-} U$ pdate $\left(G_{1} \backslash v, T_{1}, K_{1},\left\{v_{1}, v_{2}\right\}\right)$, where $\left\{v_{1}, v_{2}\right\} \subseteq V\left(G_{1}\right) \backslash$ $\left(T_{1} \cup K_{1} \cup\{v\}\right)$, then $Y(z) \leq Y(x)-3$.

3. If $\left(G_{2}, T_{2}, K_{2}\right)=\left(G_{1} \backslash v, T_{1}, K_{1}\right)$ then $Y(z) \leq Y(x)-1$.

Proof. Let $z$ be a child of $x$ associated with $\left(G_{2}, T_{2}, K_{2}\right)$ and assume that $\left(G_{2}, T_{2}, K_{2}\right)=T_{-} U p d a t e\left(G_{1}, T_{1}, K_{1}, v\right)$. Recall that all the triplets associated with the nodes of $S T$ are FCs (Lemma 6). By definition of $W$ and Lemma 1, the vertices of $W$ can be partitioned into two subsets, $W_{1} \subseteq \operatorname{Free}\left(G_{1}, T_{1}\right) \backslash K_{1}$ and $W_{2} \subseteq \operatorname{Bnd}\left(G_{1}, T_{1}\right)$.

Observe that $\left|\operatorname{Free}\left(G_{2}, T_{2}\right) \backslash K_{2}\right| \leq\left|\operatorname{Free}\left(G_{1}, T_{1}\right) \backslash K_{1}\right|-\left|W_{1}\right|$. Really, Free $\left(G_{2}, T_{2}\right) \backslash K_{2}$ are the vertices of $V\left(G_{2}\right) \backslash\left(T_{2} \cup K_{2}\right)$ that do not have neighbors in $T_{2}$. Taking into account that $T_{1} \subset T_{2}, T_{1} \cup K_{1} \subset T_{2} \cup K_{2}$, and $V\left(G_{2}\right) \subseteq V\left(G_{1}\right)$, it is clear that Free $\left(G_{2}, T_{2}\right) \backslash K_{2} \subseteq$ Free $\left(G_{1}, T_{1}\right) \backslash K_{1}$. Further, applying Lemma 3, we obtain that Free $\left(G_{2}, T_{2}\right) \backslash K_{2} \subseteq\left(\operatorname{Free}\left(G_{1}, T_{1}\right) \backslash K_{1}\right) \backslash W_{1}$. Considering that $W_{1} \subseteq \operatorname{Free}\left(G_{1}, T_{1}\right) \backslash K_{1}$, we get the desired inequality. A vertex of $W_{1}$ can be either removed from $G_{2}$ or added to $\operatorname{Bnd}\left(G_{2}, T_{2}\right)$. In the former case the value of $Y(z)$ is decreased by $c$ with respect to $Y(x)$, in the second case $Y(z)$ is decreased only by $c-1$, because the weight of the vertex is changed from $c$ to 
1. We evaluate the maximal possible weight of $Y(z)$, hence we can assume that all vertices of $W_{1}$ are moved to $\operatorname{Bnd}\left(G_{2}, T_{2}\right)$, decreasing $Y(z)$ by $(c-1)\left|W_{1}\right|$.

The vertices of $W_{2}$ are removed from $G_{2}$ by Lemma 3 and vertex $v$ is moved from $\operatorname{Bnd}\left(G_{1}, T_{1}\right)$ to $T_{2}$. These transformations decrease the value of $Y(z)$ with respect to $Y(x)$ by $\left|W_{2}\right|+1$. Combining the above argumentation, and taking into account that $c=1.565$, we see that $Y(z) \leq Y(x)-\left((c-1) *\left|W_{1}\right|+\left|W_{2}\right|+1\right) \leq$ $Y(x)-((c-1)|W|+1)$, proving the first statement.

If the condition of the second statement holds then $\mid$ Free $\left(G_{2}, T_{2}\right) \backslash K_{2} \mid+$ $\left|\operatorname{Bnd}\left(G_{2}, T_{2}\right)\right| \leq\left|\operatorname{Free}\left(G_{1}, T_{1}\right) \backslash K_{1}\right|+\left|\operatorname{Bnd}\left(G_{1}, T_{1}\right)\right|-3$ because $v$ is removed from $G_{2}, v_{1}$ and $v_{2}$ are moved to $T_{2} \cup K_{2}$. Removing of anyone of these vertices decreases $Y(z)$ by at least 1 . The second statement immediately follows.

The last statement is immediate when we observe that $\left|\operatorname{Free}\left(G_{2}, T_{2}\right) \backslash K_{2}\right|+$ $\left|\operatorname{Bnd}\left(G_{2}, T_{2}\right)\right| \leq\left|\operatorname{Free}\left(G_{1}, T_{1}\right) \backslash K_{1}\right|+\left|\operatorname{Bnd}\left(G_{1}, T_{1}\right)\right|-1$ if the condition of the last statement holds.

Let $m$ be an integer such that there is a node $x$ of $S T$ with $Y(x)=m$. We denote by $F(m)$ the maximum possible number of nodes of the subtree rooted at $x$.

Lemma 13. For any node $x$ of $S T, F(Y(x))$ is bounded by $O^{*}\left(1.50189^{Y(x)}\right)$.

Proof. Let $\left(G_{1}, T_{1}, K_{1}\right)$ be the triplet associated with $x$. Recall that $Y(x)=$ c|Free $\left(G_{1}, T_{1}\right) \backslash K_{1}|+| \operatorname{Bnd}\left(G_{1}, T_{1}\right) \mid$. Clearly, $Y(x) \geq 0$.

Assume that $Y(x)=0$. It is only possible when $V\left(G_{1}\right)=T_{1} \cup K_{1}$. According to Algorithm 1, $x$ is a leaf, hence the lemma holds for this case.

Assume now that $Y(x)>0$. Clearly, $x$ is a non-leaf. If $x$ has only one child $z$ then $z$ is necessarily associated with $T_{-} \operatorname{Update}\left(G_{1}, T_{1}, K_{1}, v\right)$ for some $v \in$ $V\left(G_{1}\right) \backslash\left(T_{1} \cup K_{1}\right)$. By Lemma $12, Y(z) \leq Y(x)-1$ (the equality holds when $v$ is not $K_{1}$-connected to any vertex of $\left.V\left(G_{1}\right) \backslash\left(T_{1} \cup K_{1} \cup\{v\}\right)\right)$. In this case, $F(Y(x))=F(Y(z))+1=F(Y(x)-l)+1$, where $l \geq 1$.

If $x$ has two children, $z_{1}$ and $z_{2}$, one of them, say $z_{1}$, is necessary associated with the triplet returned by $T_{-} U p d a t e\left(G_{1}, T_{1}, K_{1}, v\right)$. The node $z_{2}$ is associated either with $K_{-} U p d a t e\left(G_{1} \backslash v, T_{1}, K_{1},\left\{v_{1}, v_{2}\right\}\right)$ or with $\left(G_{1} \backslash v, T_{1}, K_{1}\right)$.

In the former case, $\left\{v_{1}, v_{2}\right\}$ is the set of vertices of $V\left(G_{1}\right) \backslash\left(T_{1} \cup K_{1} \cup\{v\}\right)$ that are $K_{1}$-connected to $v$. By the first part of Lemma 12, $Y\left(z_{1}\right) \leq Y(x)-(2(c-$ $1)+1)$, by the second part of the same lemma, $Y\left(z_{2}\right) \leq Y(x)-3$. Substituting $c=1.565$, we obtain $F(Y(x))=F\left(Y\left(z_{1}\right)\right)+F\left(Y\left(z_{2}\right)\right)+1=F\left(Y(x)-l_{1}\right)+$ $F\left(Y(x)-l_{2}\right)+1$, where $l_{1} \geq 2.13, l_{2} \geq 3$.

In the latter case, it follows from the description of Algorithm 1 that $v$ is $K_{1}$-connected to at least 3 vertices of $V\left(G_{1}\right) \backslash\left(T_{1} \cup K_{1} \cup\{v\}\right)$. Consequently, $Y\left(z_{1}\right) \leq Y(x)-(3(c-1)+1)$ and $Y\left(z_{2}\right) \leq Y(x)-1$ by the first and the last parts of Lemma 12. Arguing as for the previous two cases, we obtain $F(Y(x))=$ $F\left(\left(Y(x)-l_{1}\right)+F\left(Y(x)-l_{2}\right)+1\right.$, where $l_{1} \geq 2.695, l_{2} \geq 1$.

The last recursive relation for $F(Y(x))$ yields the worst upper bound. Taking into account that the upper bound is exponential, we can ignore the additive constant because it contributes only a polynomial factor to the resulting bound. The 
upper bound following from the expression $F(Y(x))=F(Y(x)-1)+F(Y(x)-$ $2.695)$ is $O^{*}\left(\beta^{Y(x)}\right)$, where $\beta$ is the largest root of the equation $\beta^{2.695}=\beta^{1.695}+1$. A simple computation shows that $1.50188<\beta<1.50189$.

Theorem 2. The Main_MIF algorithm, applied to a graph $G$ with $n$ vertices, takes $O^{*}\left(1.8899^{n}\right)$ time and a polynomial space.

Proof. Let $x$ be the root node of $S T$. It is associated with the triplet $(G, \emptyset, K)$. Then $Y(x)=c \mid$ Free $(G, \emptyset) \backslash K|+| \operatorname{Bnd}(G, \emptyset)|=c|$ Free $(G, \emptyset) \backslash K \mid \leq c n$, where $c=1.565$. It follows that $S T$ has $O^{*}\left(1.50189^{1.565 n}\right)<O^{*}\left(1.8899^{n}\right)$ nodes. The upper bound on the time-complexity of Main_MIF can be obtained by summing up the bounds on the processing time spent to every node of $S T$. Observe that processing of a node includes all the operations performed by Find_MIF except the recursive calls (whose processing time is related to other nodes of $S T$ ). The total time of these operations can be bounded by a polynomial multiplied to a number of nodes of $S T$. The resulting polynomial is suppressed by the $O^{*}$ notation, hence $O^{*}\left(1.8899^{n}\right)$ is an upper bound on the time complexity of Main_MIF.

Observe that Find_MIF has a polynomial space complexity because it is a backtrack-like procedure without explicit recording of results related to intermediate recursive calls.

\section{References}

1. J. Byskov. Enumerating maximal independent sets with applications to graph colouring. Operations Research Letters, 32(6):547-556, November 2004.

2. E. Dantsin, A. Goerdt, E. Hirsch, R. Kannan, J. Kleinberg, C. Papadimitriou, P. Raghavan, and U. Schöning. A deterministic $(2-2 /(\mathrm{k}+1))^{\mathrm{n}}$ algorithm for k-sat based on local search. Theor. Comput. Sci., 289(1):69-83, 2002.

3. D. Eppstein. Improved algorithms for 3-coloring, 3-edge coloring and constraint satisfaction. In SODA-2001, pages 329-337, 2001.

4. F. Fomin, F. Grandoni, and D. Kratsch. Measure and conquer: Domination - a case study. In ICALP, pages 191-203, 2005.

5. F. Fomin, F. Grandoni, and D. Kratsch. Some new techniques in design and analysis of exact (exponential) algorithms. Bulletin of the EATCS, 87:47-77, 2005.

6. F. Fomin and A. Pyatkin. Finding minimum feedback vertex set in bipartite graphs. In Report $N$ 291, Department of Informatics, University of Bergen, 2005.

7. R. Karp. Reducibility among combinatorial problems. In Complexity of Computer Computations, 1972.

8. V. Raman, S. Saurabh, and S. Sikdar. Improved exact exponential algorithms for vertex bipartization and other problems. In ICTCS, pages 375-389, 2005.

9. J. Robson. Algorithms for maximum independent sets. Journal of Algorithms, 7:425-440, 1986.

10. G. Woeginger. Exact algorithms for NP-hard problems: A survey. In Combinatorial Optimization: "Eureka, you shrink", LNCS 2570, pages 185-207, 2003. 